\title{
Strategies of Handling a Stenotic Unprotected Left Main Coronary Artery by Multi-Artery Fractional Flow Reserve Method
}

\author{
Ilan A Yaeger* \\ Department of Cardiology, Israel
}

*Corresponding author: Ilan A Yaeger, Department of cardiology, Israel, Email:

ilan.yaeger41@gmail.com
Received Date: January 29, 2019

Published Date: February 22, 2019

\begin{abstract}
Background and aims: By present guidelines, unprotected stenotic LMCA that requires revascularization is revascularized in most cases by a coronary artery bypass graft (CABG) operation whereas only select groups of patients that are contraindicated for CABG operation are treated by percutaneous transluminal coronary angioplasty (PTCA). A possibility of a CABG operation is never taken lightly, therefore ways of avoiding it within the LMCA-LCx-LAD stenotic configuration are explored in this article.

Methods and results: A numerical simulation of intracoronary pressures combined with the multi-artery FFR method is applied to the stenotic 3-artery configuration LMCA-LCX-LAD under conditions of stable and minimal microvascular resistance. In this method a clear distinction is made between the familiar FFR ${ }^{\text {true }}$ which is the FFR of an artery in its virtually stand-alone position and its actual FFR (denoted FFR ${ }^{\text {real }}$ ) when the artery is part of an arterial configuration in which the stenosis-stenosis interaction with other arteries impedes and reduces its blood flow (and therefore FFR ${ }^{\text {real }} \leq$ FFR $^{\text {true }}$ ). The kind of treatment that the artery needs is now determined by its FFR ${ }^{\text {real }}$ numerical value, not by its FFR ${ }^{\text {true }}$. From the initially measured intracoronary pressures the method can yield the current status of the LMCA-LCx-LAD configuration (namely FFR ${ }^{\text {real }}$ and FFR ${ }^{\text {true }}$ of each artery). Also, from the very same data, outcomes of all possible future revascularizations can be predicted. From the predicted future outcomes one can figure out if LMCA revascularization is required and also what effect would a LMCA revascularization, or lack of it, have on the current treatment decision for LCx and/or LAD arteries. The numerical examples in the article clearly show the interdependence of treatment decisions for the various arteries through inter-arterial stenosis-stenosis interactions. It turns out that in the intermediate stenosis severity range the FFR ${ }^{\text {true }}$ of an artery may be satisfactory but its FFR ${ }^{\text {real }}$ is sometimes lower by about 0.3 indicating in most of such cases that revascularization is mandatory.
\end{abstract}

Conclusion: The first step in the multi-artery FFR method is to measure the intracoronary pressures at particular locations in the arterial configuration. The intracoronary pressures yield the present status of the configuration as well as the outcomes of all possible future revascularizations of the arteries of the configuration (provided that no revascularization-induced stenosis anatomy changes take place). This unique property yields eventually the optimal resolution of the stenotic LMCA-LCx-LAD configuration.

Keywords: Fractional flow reserve; Multi-artery fractional flow reserve; Percutaneous coronary intervention

\section{Introduction}

The LMCA is the most important coronary artery since it provides the blood supply to almost the entire left ventricle [1]. The LMCA was first assessed by an angiographic method which was problematic because of inherent difficulties associated with LMCA like short vessel segment, lack of a reference vessel, eccentricity and other unfavourable phenomena [2]. Assessment of LMCA was also made extensively by IntraVascular UltraSound (IVUS) for considerable period of time [3]. In many cases the LMCA has a particular distribution and nature of plaque. LMCA plaque was found to be diffuse and to extend into the proximal LAD, LCx arteries in most cases [4]. This undoubtedly complicates the treatment of stenotic LMCA. The results and consequences of the first percutaneous interventions in LMCA showed that at that time percutaneous transluminal coronary angioplasty (PTCA) could not be acceptable as a standard treatment [5]. This led to the consensus that PTCA is contraindicated for treating a stenotic unprotected LMCA (namely without even one bypass graft to LAD and/or LCx arteries) [6]. In view of the progress in the percutaneous coronary 
intervention (PCI) practice, in terms of technology and operators experience in recent years, there is an increasing number of reports about LMCA treatment by PCI procedures and CABG surgeries with comparable results $[7,8]$. There is no intention to participate in this debate in the present article, its aim is just to stress the importance of the LMCA revascularization issue and show some alternative options.

In recent years some researchers have realized that LMCA cannot be treated as a single and independent entity because of the effect that stenotic LAD and LCX, that are sizable arteries, may have on the blood flow of LMCA. Attempts have been made to use the basic FFR method to this end [9-11]. The basic FFR method however is a single-artery FFR method that can assess a single stenosis in a single artery [12] as well as serial stenoses within a single artery [13] but since it does not take into account stenosis-stenosis interarterial interactions, it cannot provide adequate assessment of stenosis severity in LMCA in the presence of another sizable stenotic artery. Even in the well-known FAME study [14] cases with diseased LMCA were excluded thus exposing each of LAD and LCx arteries to proximal aortic pressure making them independent of each other. Consequently, each of the so-called multi-vessel disease (MVD) cases of FAME was actually a single or a couple of independent single-vessel disease (SVD) cases and each was treated as such. The attempts to assess LMCA by the basic FFR method and figure out the effect of downstream stenoses [9-11] were dubious. In those studies, one of the downstream arteries (LAD or LCX) was stenosisfree and the other one was stenotic. The pressure wire was placed in the non-stenotic artery and the researchers claimed that practically there was no effect by downstream stenosis on FFR(LMCA) unless the downstream stenosis was proximal and severe [9-11].

In 2015 the multi-artery FFR method was developed to be used with numerical simulation of intracoronary pressures [15] in order to handle a system of two or more interacting stenotic arteries under conditions of minimal and stable microvascular resistance (either inherent or pharmacologically induced). A clear distinction was made between the familiar $\mathrm{FFR}^{\text {true }}$ and the actual FFR (denoted FFR ${ }^{\text {real }}$ ). FFR ${ }^{\text {true }}$ of a stenotic artery in the LMCALCx-LAD configuration is the ratio of the maximum flow through the stenotic artery, assuming that all other arteries are stenosisfree, and the maximal flow through the artery when all arteries of the configuration (including the artery under consideration) are virtually stenosis-free. On the other hand, FFR ${ }^{\text {real }}$ of an artery in the arterial configuration is the ratio between the actual flow through the artery when all arteries of the configuration are in their real state (some or all of which may be stenotic and interacting with the artery under consideration) and the maximum flow through the artery when all arteries of the configuration (including the one under consideration) are hypothetically stenosis-free. The kind of treatment that the artery needs is now determined by its FFR ${ }^{\text {real }}$ numerical value, not by its $\mathrm{FFR}^{\text {true }}$. The reason for this is that being a member of an arterial configuration, the artery is no longer just by itself, its flow is affected also by the flow in other arteries of the configuration (some of which may be stenotic). This effect is termed stenosis-stenosis interaction and FFR real $\leq \mathrm{FFR}^{\text {true }}$. FFR ${ }^{\text {true }}$ however is an intrinsic property of the artery. If the artery is fully revascularized, $\mathrm{FFR}^{\text {true }}$ of the artery is taken to be $\mathrm{FFR}^{\text {true }}=1.00$ (in reality it may be somewhat less). Studying the effect of downstream stenoses on LMCA by the multi-artery FFR method, it can be seen from the simulation of [15] that under the arterial conditions of [9-11] (stenotic LAD and non-stenotic LCx), in the intermediate stenosis severity range of $\mathrm{FFR}^{\text {true }}$ (LMCA) the numerical values of FFR $^{\text {real }}$ (LMCA) within that table are significantly affected by the stenotic LAD artery in its intermediate and high stenosis severity (low FFR ${ }^{\text {true }}(\mathrm{LAD})$ ) ranges.

In the present article the LMCA is assessed by the multi-artery FFR method within the 3-artery configuration LMCA-LCx-LAD in which all 3 members of the configuration may be stenotic and inter-arterial stenosis-stenosis interactions might be substantial. The aim of the article is to devise an optimal revascularization strategy of the configuration arteries in an attempt to avoid revascularization of LMCA, when possible. If such revascularization is unavoidable, and therefore carried out, it may indirectly improve also the status of one or both downstream arteries to the extent of removing the necessity for their revascularization. The difference between FFR $^{\text {true }}$ and FFR $^{\text {real }}$ of each artery reflects the intensity of the inter-arterial stenosis-stenosis interaction. Using the multiartery FFR method, if no revascularization-induced morphological plaque changes take place, a detailed assessment of the outcomes of potential revascularizations can be obtained already from the initial measurement of intracoronary pressures at some particular locations within the LMCA-LCX-LAD configuration Figure1. For simplicity, it will be assumed that collaterals are not involved and that the stenoses in all 3 arteries of the configuration in Figure 1 are localized. Although it is usually true for LAD and LCX arteries, this is usually not the case for the LMCA for which the disease may be diffuse and in many cases the LMCA plaque extends to the proximal LAD and/or LCx [4]. In order to compensate for this phenomenon, appropriate simple approximations will be made.

\section{Methods}

\section{The basic formulas of the multi-artery FFR method}

In this article a numerical simulation of intracoronary pressures is combined with the multi-artery FFR method. The method will be applied to the stenotic 3-artery configuration LMCA-LCX-LAD presented schematically in Figure 1. It is a configuration of sizable stenotic arteries of the kind that has been treated in a generalized way by the multi-artery FFR method [16]. In the present article however the focus is on the LMCA revascularization issue. Due to lack of space, the lengthy mathematics involved in the derivation of the formulas and expressions of this method will not be repeated here, the reader will rather be directed to the relevant reference when appropriate. Despite the elaborate mathematics, the final mathematical formulas are relatively simple. They will be given in the next section in a concise form and can be therefore used in real time by the practitioner in the percutaneous coronary intervention 
(PCI) process. For the convenience of the reader, the relevant notations and expressions will be given in the following Figure 1:

Artery 1 (LMCA) - functionally the epicardial conductance artery of the configuration Artery 2 (LCx) and Artery 3 (LAD) - epicardial end arteries of the configuration leading to the myocardium $\mathrm{R}_{\mathrm{it}}$ resistance of artery $i=1,2,3$; subscript $t$ indicates type of resistance (s - stenotic, mv -microvascular).

As indicated in the 1. Introduction paragraph, treatment criteria for an artery no longer relate to its FFR ${ }^{\text {true }}$, rather to its FFR ${ }^{\text {real }}$. The FFR $^{\text {true }}$ expressions however do represent intrinsic properties of the stenotic arteries. They will be therefore given in the following [17] Figure 1:

$$
\begin{aligned}
& \operatorname{FFR}^{\text {true }}(1)=1 /\left[\left(\mathrm{R}_{1 \mathrm{~s}} / \mathrm{R}_{2 \mathrm{mv}}\right)+\left(\mathrm{R}_{1 \mathrm{~s}} / \mathrm{R}_{3 \mathrm{mv}}\right)+1\right](1) \\
& \operatorname{FFR}^{\text {true }}(2)=1 /\left[1+\left(\mathrm{R}_{2 \mathrm{~s}} / \mathrm{R}_{2 \mathrm{mv}}\right)\right](2) \\
& \operatorname{FFR}^{\text {true }}(3)=1 /\left[1+\left(\mathrm{R}_{3 \mathrm{~s}} / \mathrm{R}_{3 \mathrm{mv}}\right)\right](3)
\end{aligned}
$$

Another expression that involves resistances is the ratio $\delta$ of microvascular resistances:

$$
\delta=\mathrm{R}_{3 \mathrm{mv}} / \mathrm{R}_{2 \mathrm{mv}}(4)
$$

FFR $^{\text {real }}$ of each one of the arteries of the configuration can be expressed in terms of FFR ${ }^{\text {true }}(1), \mathrm{FFR}^{\text {true }}(2), \mathrm{FFR}^{\text {true }}(3)$ and $\delta$ that can be all regarded for practical purposes as auxiliary variables $[15,16]$.

Since coronary arteries vary from person to person, so do their associated microvascular resistances and so does the ratio $\delta$. It has been shown that $\delta$ is also equal to the geometry-dependent ratio of the viscous resistances (by the Poiseuille formula) of the stems of Artery 3 (LAD) and Artery 2 (LCX) [18]. Within the statistical range of morphological variations (radii, rates of tapering and lengths) of LAD and LCx [17] in most cases $\delta$ is within close proximity of $\delta$ $=1$. Within this range of $\delta$ however the calculated $\mathrm{FFR}^{\text {real }}$ values of the arteries of the configuration exhibit low and quite acceptable variations of just $\Delta \mathrm{FFR}^{\text {real }}= \pm 0.02$, in the low and intermediate FFR stenosis severity ranges [18]. For practical reasons it is preferable to use a single value of $\delta$ in the formulas and if one chooses to use $\delta$ $=1$, the variation of $\delta$ from person to person will have little effect on FFR $^{\text {real }}$ of the arteries of the LMCA-LCX-LAD configuration since in the close proximity of $\delta=1$ the calculated $\mathrm{FFR}^{\text {real }}$ of the arteries (as functions of $\delta$ ) are statistically nearly constant within the patient population.

\section{The final concise formulas of the multi-artery FFR method for the use of the PCI practitioner}

As indicated, the $\delta$ in the expressions in this section is taken as $\delta$ $=1$. The artery indices 1, 2 and 3 in the expressions here correspond to LMCA, LCx and LAD respectively Figure 1. The initial values of the FFR ${ }^{\text {true }}$ of each one of the arteries of the configuration of Figure 1 can be obtained from the measured intracoronary pressures by the following expressions [16]:

$$
\begin{aligned}
& \mathrm{FFR}^{\text {true }}(1)=1 /\left\{\left[\left(\mathrm{P}_{\mathrm{a}}-\mathrm{P}_{\mathrm{p}}\right) \cdot(1+\delta)\right] /\left(\mathrm{P}_{3 \mathrm{~d}}+\delta \cdot \mathrm{P}_{2 \mathrm{~d}}\right)+1\right\}(5) \\
& \mathrm{FFR}^{\text {true }}(2)=\mathrm{P}_{2 \mathrm{~d}} / \mathrm{P}_{\mathrm{p}}(6)
\end{aligned}
$$

$$
\operatorname{FFR}^{\text {true }}(3)=\mathrm{P}_{3 \mathrm{~d}} / \mathrm{P}_{\mathrm{p}}(7)
$$

It should be noted that if the plaque of LMCA extends into the proximal LCx and/or LAD arteries (see 1. Introduction), $\mathrm{P}_{\mathrm{p}}$ can no longer be taken as an intracoronary pressure at one particular location and appropriate compensatory approximations need to be made. In this context, the following definitions are made Figure 1 :

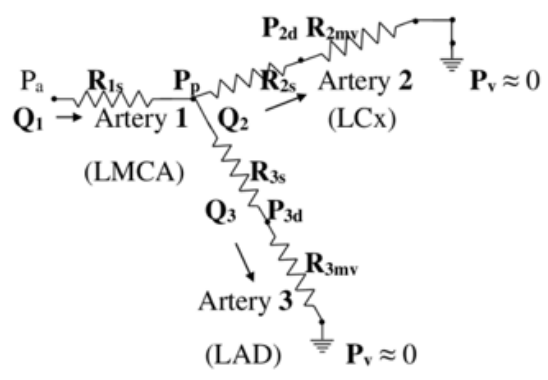

3-artery configuration pressure-Resistance-Flow scheme

Figure 1: First subscript of resistance Rit indicates the artery number $(i=1,2,3)$ and the second subscript indicates the type of resistance ( $\mathrm{t}=\mathrm{s}$-stenotic, mv-microvascular). $\mathrm{Pa}$ and $\mathrm{Pv}$ are the mean aortic pressure and the nearly zero pressure of the venous bed respectively. Pp is the proximal pressure of Artery 2 (LCx) and Artery 3 (LAD) while P2d and P3d are the distal pressures of Arteries 2 (LCX) and 3 (LAD) respectively. Qi is the blood flow (volume units per unit of time) in Artery $i(i=1,2,3)$. In the analysis of the MVD scenario in the text, Artery 1 (LMCA) is functionally the conductance artery whereas Artery 2 (LCx) and Artery 3 (LAD) are the end arteries that lead to the myocardium.

$\mathrm{P}_{2 p}$ - intracoronary pressure in proximal Artery 2 (LCx) measured at a point immediately following LMCA plaque and preceding $\mathrm{R}_{2 \mathrm{~s}}$

$\mathrm{P}_{3 p}$ - intracoronary pressure in proximal Artery 3 (LAD) measured at a point immediately following LMCA plaque and preceding $\mathrm{R}_{3 \mathrm{~s}}$

In such a case the $\mathrm{P}_{\mathrm{p}}$ in the formulas in this article is defined as the following average:

$\mathrm{P}_{\mathrm{p}}=\left(\mathrm{P}_{2 \mathrm{p}}+\mathrm{P}_{3 \mathrm{p}}\right) / 2(8)$

The current values of the FFR real of the arteries of the configuration in Figure 1 can be also obtained from the measured intracoronary pressures [16]:

$$
\begin{aligned}
& \operatorname{FFR}^{\text {real }}(1)=\left(\mathrm{P}_{3 \mathrm{~d}}+\delta \cdot \mathrm{P}_{2 \mathrm{~d}}\right) /\left[\mathrm{P}_{\mathrm{a}} \cdot(1+\delta)\right](9) \\
& \operatorname{FFR}^{\text {real }}(2)=\mathrm{P}_{2 \mathrm{~d}} / \mathrm{P}_{\mathrm{a}}(10) \\
& \operatorname{FFR}^{\text {real }}(3)=\mathrm{P}_{3 \mathrm{~d}} / \mathrm{P}_{\mathrm{a}}(11)
\end{aligned}
$$

From the current $\mathrm{FFR}^{\text {real }}$ values it can be determined if revascularizations are needed. In order to predict outcomes of potential revascularizations, FFR ${ }^{\text {real }}$ of each artery in Figure 1 needs to be expressed in terms of $\mathrm{FFR}^{\text {true }}$ of each of the arteries and $\delta$. One has [16]:

$$
\begin{aligned}
& \operatorname{FFR}^{\text {real }}(1)=\left\{\operatorname{FFR}^{\text {true }}(1) \cdot\left[\mathrm{FFR}^{\text {true }}(3)+\delta \cdot \mathrm{FFR}^{\text {true }}(2)\right]\right\} / \\
& \left\{\left[1-\mathrm{FFR}^{\text {true }}(1)\right] \cdot\left[\delta \cdot \mathrm{FFR}^{\text {true }}(2)+\mathrm{FFR}^{\text {true }}(3)\right]+(1+\delta) \cdot \mathrm{FFR}^{\text {tru }}\right.
\end{aligned}
$$


(13)

$$
\begin{aligned}
& \mathrm{FFR}^{\text {real }}(2)=\left[(1+\delta) \cdot \mathrm{FFR}^{\text {true }}(1) \cdot \mathrm{FFR}^{\text {true }}(2)\right] / \\
& \left\{\left[1-\mathrm{FFR}^{\text {true }}(1)\right] \cdot\left[\delta \cdot \mathrm{FFR}^{\text {true }}(2)+\mathrm{FFR}^{\text {true }}(3)\right]+(1+\delta) \cdot \mathrm{FFR}^{\text {true }}(1)\right\}
\end{aligned}
$$

$$
\operatorname{FFR}^{\text {real }}(3)=\left[(1+\delta) \cdot \mathrm{FFR}^{\text {true }}(1) \cdot \mathrm{FFR}^{\text {true }}(3)\right] /
$$

$$
\left\{\left[1-\mathrm{FFR}^{\text {true }}(1)\right] \cdot\left[\delta \cdot \mathrm{FFR}^{\text {true }}(2)+\mathrm{FFR}^{\text {true }}(3)\right]+(1+\delta) \cdot \mathrm{FFR}^{\text {true }}(1)\right\}
$$

If an $\mathrm{FFR}^{\text {real }}$ of each artery is to be predicted from expressions (12), (13) and (14) in case of a planned revascularization, the $F_{F} R^{\text {true }}$ for the artery to be revascularized is taken as $\mathrm{FFR}^{\text {true }}=1.00$ whereas FFR $^{\text {true }}$ values of the other arteries remain at their initial value (see numerical Example \#1 and Example \#2 in next paragraph). Note that all three FFR ${ }^{\text {real }}$ expressions (12), (13) and (14) have the same denominator and this facilitates and shortens the data processing time in the PCI procedure.

\section{Result}

In this paragraph strategies will be devised to handle a stenotic LMCA by the multi-artery FFR method. Appropriate numerical examples will be presented with intracoronary pressure values at particular locations within the LMCA-LCX-LAD configuration Figure 1 given by numerical simulation.

\section{Example \#1:}

In this example the simulated intracoronary pressures are taken to be the following Figure 1:

$$
\begin{aligned}
& \mathrm{P}_{\mathrm{a}}=105 \mathrm{mmHg} \\
& \mathrm{P}_{\mathrm{p}}=86 \mathrm{mmHg} \\
& \mathrm{P}_{2 \mathrm{~d}}=75 \mathrm{mmHg} \\
& \mathrm{P}_{3 \mathrm{~d}}=80 \mathrm{mmHg}
\end{aligned}
$$

$\delta$ is taken to be $\delta=1$

The artery indices 1, 2 and 3 correspond to LMCA, LCx and LAD respectively.

The calculated current $\mathrm{FFR}^{\text {true }}$ numerical values of the arteries of Figure 1then are:

$$
\begin{aligned}
& \operatorname{FFR}^{\text {true }}(1)=0.80(\text { see expression }(5)) \\
& \operatorname{FFR}^{\text {true }}(2)=0.87(\text { see expression }(6)) \\
& \operatorname{FFR}^{\text {true }}(3)=0.93(\text { see expression (7) })
\end{aligned}
$$

The corresponding FFR ${ }^{\text {real }}$ numerical values are:

$$
\begin{aligned}
& F_{F R}^{\text {real }}(1)=0.74(\text { see expression }(9)) \\
& F^{\text {real }}(2)=0.71(\text { see expression }(10)) \\
& \operatorname{FFR}^{\text {real }}(3)=0.76(\text { see expression }(11))
\end{aligned}
$$

It is evident that FFR ${ }^{\text {real }}$ of Artery 1 and Artery Figure 1 are unacceptable whereas FFR ${ }^{\text {real }}(3)$ is barely within the FFR treatment decision 'grey zone'. Some revascularization options will be now tried:

Option \#1 - Revascularization of Artery $2-\mathrm{FFR}^{\text {true }}(2)=1.00$
The FFR ${ }^{\text {true }}$ values of the other two arteries remain equal to their current values:

$$
\operatorname{FFR}^{\text {true }}(1)=0.80 ; \operatorname{FFR}^{\text {true }}(3)=0.93 ;
$$

Using these values, post revascularization $\mathrm{FFR}^{\text {real }}$ values of each artery can be predicted:

$\mathrm{FFR}^{\text {real }}(1)=0.78($ see expression $(12))$

$\operatorname{FFR}^{\text {real }}(2)=0.81($ see expression $(13))$

$\operatorname{FFR}^{\text {real }}(3)=0.75$ (see expression (14) )

Note that when LMCA is stenotic, the blood flows in LAD and LCx are competing. Revascularization of LCx Figure 1 increases the flow in LCx artery but reduces the flow in LAD artery. This is why the revascularization would result in slight reduction of $\mathrm{FFR}^{\text {real }}(3)$ (from 0.76 to 0.75 ).

The FFR ${ }^{\text {real }}$ values of the arteries are within the FFR treatment decision 'grey zone' and it is up to the practitioner to take into account the clinical condition of the particular patient and decide whether such a post-revascularization outcome might be satisfactory or not. Note that if both Artery 2 and Artery 3 Figure 1 are revascularized, not just Artery 2, then by definition one would have $\mathrm{FFR}^{\text {real }}(1)=\mathrm{FFR}^{\text {true }}(1)=0.80$. Namely not much would be gained for LMCA by revascularizing one more end artery in this case. However, depending on the clinical condition of the particular patient, it is up to the PCI practitioner to make a decision if it would be right to revascularize LMCA at a cost of subjecting the patient to stenting or a full scale CABG operation.

Now let's see Figure 1 what can be gained if the PCI practitioner decides to have LMCA revascularized:

Option \#2 - Revascularization of Artery 1 - FFR ${ }^{\text {true }}(1)=1.00$

The FFR ${ }^{\text {true }}$ values of Artery 2 and Artery 3 remain equal to their current values:

$$
\operatorname{FFR}^{\text {true }}(2)=0.87 ; \operatorname{FFR}^{\text {true }}(3)=0.93 ;
$$

The post-revascularization predicted values of $\mathrm{FFR}^{\text {real }}$ of the arteries of the configuration are:

$$
\begin{aligned}
& \operatorname{FFR}^{\text {real }}(1)=0.90(\text { see expression }(12)) \\
& \operatorname{FFR}^{\text {real }}(2)=0.87(\text { see expression }(13)) \\
& \operatorname{FFR}^{\text {real }}(3)=0.93(\text { see expression }(14))
\end{aligned}
$$

The FFR ${ }^{\text {real }}$ values of all arteries are acceptable. Therefore, if the PCI practitioner intends to have LMCA revascularized, there is no need to revascularize any of the end Arteries 2 and 3 Figure 1.

\section{Example \#2:}

The simulated intracoronary pressures are taken to be the following Figure 1:

$$
\begin{aligned}
& P_{a}=102 \mathrm{mmHg} \\
& P_{2 p}=72 \mathrm{mmHg} \\
& P_{3 p}=68 \mathrm{mmHg}
\end{aligned}
$$


$\mathrm{P}_{\mathrm{p}}=\left(\mathrm{P}_{2 \mathrm{p}}+\mathrm{P}_{3 \mathrm{p}}\right) / 2=70 \mathrm{mmHg}$

$\mathrm{P}_{2 \mathrm{~d}}=50 \mathrm{mmHg}$

$\mathrm{P}_{3 \mathrm{~d}}=63 \mathrm{mmHg}$

One takes $\delta$ to be $\delta=1$

The artery indices 1, 2 and 3 correspond to LMCA, LCx and LAD respectively.

The calculated current FFR ${ }^{\text {true }}$ numerical values of the Arteries 1, 2, and 3 of Figure 1 are:

$$
\begin{aligned}
& \operatorname{FFR}^{\text {true }}(1)=0.64(\text { see expression (5) }) \\
& \operatorname{FFR}^{\text {true }}(2)=0.71(\text { see expression }(6)) \\
& \operatorname{FFR}^{\text {true }}(3)=0.90(\text { see expression }(7)) \\
& \operatorname{The~FFR~}^{\text {real }} \text { numerical values are: } \\
& \operatorname{FFR}^{\text {real }}(1)=0.55(\text { see expression (9)) } \\
& \operatorname{FFR}^{\text {real }}(2)=0.49(\text { see expression }(10)) \\
& \operatorname{FFR}^{\text {real }}(3)=0.62(\text { see expression }(11))
\end{aligned}
$$

It is obvious that the current FFR $^{\text {real }}$ values are unacceptable and that the revascularization options for improving the condition of LMCA are quite limited.

Option \#1: If both arteries LCx and LAD are revascularized, by definition one has

$\operatorname{FFR}^{\text {real }}(1)=\operatorname{FFR}^{\text {true }}(1)=0.64$

This is still unacceptable, therefore the revascularization of LMCA is inevitable. In such a case

$\operatorname{FFR}^{\text {true }}(1)=1.00$ while Arteries 2 and 3 Figure 1 maintain their current FFR ${ }^{\text {true }}$ values:

$$
\begin{aligned}
& \operatorname{FFR}^{\text {true }}(2)=0.71(\text { see expression }(6)) \\
& \operatorname{FFR}^{\text {true }}(3)=0.90(\text { see expression }(7))
\end{aligned}
$$

Calculating the $\mathrm{FFR}^{\text {real }}$ of the arteries following LMCA revascularization, one has

$$
\begin{aligned}
& \operatorname{FFR}^{\text {real }}(1)=0.81(\text { see expression }(12)) \\
& \operatorname{FFR}^{\text {real }}(2)=0.71(\text { see expression }(13)) \\
& \operatorname{FFR}^{\text {real }}(3)=0.90(\text { see expression }(14))
\end{aligned}
$$

The revascularization of LMCA may have elevated FFR $^{\text {real }}(1)$ to an acceptable level by the PCI practitioner but FFR ${ }^{\text {real }}(2)$ is still unacceptable. There is no alternative but to revascularize also Artery 2 . Such a revascularization may improve not only $\mathrm{FFR}^{\text {real }}(2)$ but further improve FFR ${ }^{\text {real }}(1)$ due to stenosis-stenosis interaction.

Let's check therefore Option \#2 of revascularizing both Artery 1 and Artery 2:

$$
\begin{aligned}
& \operatorname{FFR}^{\text {true }}(1)=\operatorname{FFR}^{\text {true }}(2)=1.00 \operatorname{FFR}^{\text {true }}(3)=0.90 \\
& \text { Option \#2 yields: }
\end{aligned}
$$

$\mathrm{FFR}^{\text {real }}(1)=0.95($ see expression $(12))$
$\operatorname{FFR}^{\text {real }}(2)=1.00($ see expression (13) $)$

$\mathrm{FFR}^{\text {real }}(3)=0.90($ see expression $(14))$

All these FFR $^{\text {real }}$ values are acceptable.

As expected, revascularization of both Artery 1 and Artery 2 has improved $\mathrm{FFR}^{\text {real }}(1)$ as well as $\mathrm{FFR}^{\text {real }}(2)$. Option \#2 is therefore the optimal resolution of the stenotic 3-artery configuration Figure 1 in this case.

\section{Discussion and Conclusion}

\section{Uniqueness of the combined numerical simulation and multi-artery FFR method}

For more than two decades, one of the major courses of activity in cardiology has been the study of hemodynamic effects in human coronary arteries by simulation methods prior to progressing to the clinical level. The effect of stenosis in coronary arteries has been studied particularly by mechanical simulation in vitro methods [19] as well as by porcine model in vivo simulation methods [20]. Unlike those simulations, the numerical simulation of intracoronary pressures combined with the multi-artery FFR method provides a natural and smooth transition to the process that takes actually place in the catheterization laboratory. It should be also noted that the simulated intracoronary pressures used in the article belong to the numerical range encountered in the real PCI practice. Therefore, the PCI practitioner can simply use the measured pressure values readily with the formulas and expressions given in the article. In order to demonstrate this, in this paragraph the logic and particulars of the two examples presented in the article will be discussed first and discussion of other related matters will follow.

\section{A step-by-step walk through Examples \#1 and \#2}

Due to the extreme importance of LMCA, in the stenosis severity assessment of the arteries of the LMCA-LCX-LAD configuration, the primary attention of the PCI practitioner should focus on LMCA. One should figure out firstly if LMCA revascularization is mandatory in the case under consideration. In this paragraph it will be exactly described what should be systematically done to this end, employing the multi-artery FFR method. At the very beginning the practitioner should figure out what is the possible maximal FFR $^{\text {real }}$ (LMCA). The maximal level of FFR ${ }^{\text {real }}(\mathrm{LMCA})$ is obtained by revascularizing both $\mathrm{LCX}$ and LAD, namely when $\mathrm{FFR}^{\text {real }}(\mathrm{LMCA})=$ FFR $^{\text {true }}$ (LMCA). This maximal level needs to be checked in order to see if its numerical value is below the numerical 'grey zone' lower limit of the FFR treatment decision range. A maximal FFR ${ }^{\text {real }}$ (LMCA) below the lower limit of the 'grey zone' would imply a mandatory revascularization of LMCA. In Example \#2 of paragraph 3. Results a description of such a case is presented. One has the following relationship: maximal $\mathrm{FFR}^{\text {real }}(\mathrm{LMCA})=\mathrm{FFR}^{\text {true }}(\mathrm{LMCA})=\mathrm{FFR}^{\text {true }}(1)$ $=1 /\left\{\left[\left(\mathrm{P}_{\mathrm{a}}-\mathrm{P}_{\mathrm{p}}\right) \cdot(1+\delta)\right] /\left(\mathbf{P}_{3 \mathrm{~d}}+\delta \cdot \mathbf{P}_{2 \mathrm{~d}}\right)+1\right\}$ with $\delta=1$ Figure 1 . Note that should a revascularization of LMCA take place, the $\mathrm{FFR}^{\text {real }}$ of all the arteries would change, therefore the outcome of LMCA revascularization should be predicted prior to any further future action with regard to other arteries (see expressions (12), (13) and (14) in paragraph 2. Methods). From the initial values of 
the FFR ${ }^{\text {real }}$ of all the arteries in Example \#2, it seemed that all 3 arteries would need revascularization. However from the predicted outcome of LMCA revascularization it could be seen that it would bring sufficient (though not maximal) relief to Artery 1 (LMCA) $\left(F_{F R}{ }^{\text {real }}(1)=0.81\right)$ and to Artery $3(\mathrm{LAD})\left(\mathrm{FFR}^{\text {real }}(3)=0.90\right)$ but not to Artery $2(\mathrm{LCX})\left(\mathrm{FFR}^{\text {real }}(2)=0.71\right)$. Therefore Artery 2 needs to be revascularized too. When Artery $\mathbf{2}$ is revascularized (in addition to LMCA) the predicted outcome would be $\mathrm{FFR}^{\text {real }}(\mathrm{LMCA})=0.95$ , $F_{F R}{ }^{\text {real }}(\mathrm{LCX})=1.00$ and $\mathrm{FFR}^{\text {real }}(\mathrm{LAD})=0.90$ which is the optimal resolution of the configuration Figure 1. Note that the improvement of $\mathrm{FFR}^{\text {real }}(\mathrm{LMCA})$ (from $\mathrm{FFR}^{\text {real }}(\mathrm{LMCA})=0.81$ to $\mathrm{FFR}^{\text {real }}(\mathrm{LMCA})=$ 0.95 ) is due to the inter-arterial stenosis-stenosis interaction.

Now let's consider a case in which LMCA revascularization may be avoidable, depending on the clinical considerations of the PCI practitioner. Such a case is described in Example \#1 of paragraph 3. Results Figure 1. The FFR ${ }^{\text {real }}$ values of all 3 arteries of the configuration in that example are very close to the lower end of the 'grey zone' of the FFR decision range, slightly above or below the lower end. Excluding the option of revascularizing LMCA, in an attempt to elevate the $\mathrm{FFR}^{\text {real }}$ of the arteries, one of the end arteries needs to be revascularized. In Example \#1 initially one has $F_{F R}^{\text {true }}(L C x)=0.87$ and $F_{F R}{ }^{\text {true }}(L A D)=0.93$. Since revascularization yields $\mathrm{FFR}^{\text {true }}=1.00$ for a fully revascularized artery then with FFR $^{\text {true }}\left(\mathrm{LCX}\right.$ ) being farther away from 1.00 than $\mathrm{FFR}^{\text {true }}(\mathrm{LAD})$, it seems that it would be more beneficial to revascularize Artery 2 (LCx) than Artery 3 (LAD). Revascularization of Artery 2 (LCx) yields:

$\operatorname{FFR}^{\text {real }}(\mathrm{LMCA})=0.78 ; \mathrm{FFR}^{\text {real }}(\mathrm{LCx})=0.81 ; \mathrm{FFR}^{\text {real }}(\mathrm{LAD})=0.75 ;$

The net gain of such a revascularization is that the FFR $^{\text {real }}$ values are essentially elevated and pushed farther up into the FFR treatment decision 'grey zone', which makes the condition of the arteries barely acceptable. Note that when LMCA is stenotic, the flows in LAD (Artery 3) and CLx (Artery 2) compete and an increase of the CLx flow results in decrease of the LAD flow. This is why there is a slight reduction in FFR ${ }^{\text {real }}$ (LAD) (from 0.76 to 0.75 ). As indicated, since FFR ${ }^{\text {true }}$ (LAD) is already very close to 1.00 , no significant change of $\mathrm{FFR}^{\text {real }}$ values of the arteries would result by revascularizing Artery 3 (LAD) Figure 1 . Should the PCI practitioner decide to revascularize LMCA, the FFR ${ }^{\text {real }}$ of all arteries would be acceptable by a wide margin (see end of Example \#1 in paragraph 3. Results).

In this section some strategies of systematically resolving stenotic LMCA within stenotic LMCA-LCx-LAD configurations by the multi-artery FFR method have been presented. One of the important distinctions between an unprotected stenotic LMCA and other stenotic coronary arteries is the way of treatment. If a revascularization of an unprotected LMCA is required, the PCI practitioner has to turn to the current clinical practice guidelines [2] in order to make the choice between a CABG operation and a PTCA procedure. Potential revascularization of LMCA, if carried out, may affect treatment decisions with regard to LCX and/or LAD arteries. This is why the possibility of LMCA revascularization should be always explored first in the assessment of a stenotic LMCA-LCX-LAD configuration.

\section{The inter-arterial stenosis-stenosis interaction}

The present article also sheds new light on the concept of the elusive stenosis-stenosis interaction. This concept came into being about 20 years ago when it was realized that the interaction between 2 stenoses in a single artery is such that FFR of each stenotic segment separately cannot be calculated by the simple equation for isolated stenoses [21]. In the present article the concept of single-artery stenosis-stenosis interaction is extended to inter-arterial stenosis-stenosis interaction. Being a physical effect, it should be possible to provide a quantitative measure of its intensity. The intensity of this interaction, the extent to which the flows in other arteries of the configuration affect the flow in an artery, is reflected by the magnitude of the difference $\Delta F F R$ between its $F_{F R}{ }^{\text {true }}$ and its $F_{F R}{ }^{\text {real }}$, namely $\triangle F F R=F^{\text {true }}-F^{2} R^{\text {real }} . \Delta F F R$ can be therefore regarded as the most natural choice of a quantitative measure of the intensity of the stenosis-stenosis interaction. In the numerical examples presented in the article it is shown that in the intermediate stenosis severity range $\Delta \mathrm{FFR}$ of an artery may reach even a value as high as 0.3 which is about 3 -fold the width of the FFR treatment decision 'grey zone'.

\section{Some downsides of the multi-artery FFR method}

Being a relatively novel method, the multi-artery FFR method deals essentially with the salient features of stenotic coronary 3 -artery configurations $[15,16,18]$. On the grounds of simplicity, it is assumed in the present article that collaterals are not involved with the arteries of the stenotic coronary configuration. At an advanced stage of coronary disease however, collaterals may assume a substantially significant role.

From the analysis of the possibilities of resolving the stenotic LMCA-LCX-LAD configurations in Example \#1 and Example \#2 of this article (in 3. Results) some downsides of the multi-artery FFR method stand out. Firstly, the method does not (and actually cannot) take into account the exact location of stenoses. Assuming a same FFR $^{\text {real }}$, a stenosis in the proximal section of an artery potentially puts at risk more side branches than a stenosis in the distal section of an artery. Such a matter can be taken into consideration only by a PCI practitioner who can weigh the importance of such a state of a configuration. Secondly, more often than not, the PCI practitioner may be confronted with an unfavourable numerical value of FFR ${ }^{\text {real }}$ of an artery. If FFR ${ }^{\text {real }}$ is below the lower end of the 'grey zone' of the FFR decision range then it is undisputedly not acceptable. However, if $\mathrm{FFR}^{\text {real }}$ is within the 'grey zone' or slightly above it, the PCI practitioner has to decide what can be regarded 'safe' for the patient under consideration and what would be a clinically justifiable 'price' (a CABG operation or PTCA) for it. In conclusion, although the multi-artery FFR method provides the PCI practitioner with precise data about the status of the LMCA-LCXLAD configuration (namely $\mathrm{FFR}^{\text {real }}$ and $\mathrm{FFR}^{\text {true }}$ of each artery), in order to devise a strategy of handling a complex stenotic coronary configuration, especially one that involves an unprotected LMCA, 
professional experience and careful judgement on the part of the PCI practitioner are virtually irreplaceable.

\section{Acknowledgement}

None.

\section{Conflict of Interest}

No conflict of interest.

\section{References}

1. Hamilos M, Muller O, De Bruyne B (2010) The left main coronary artery deserves more than a quick look. Interv Cardiol 2: 7-11.

2. Ramadan R, Boden WE, Kinlay S (2018) Management of Left Main Coronary Arter Disease. J Am Heart Assoc 7: e008151.

3. Das P, Meredith I (2007) Role of intravascular ultrasound in unprotected left main percutaneous coronary intervention. Expert Rev Cardiovasc Ther 5: 81-89.

4. Oviedo C, Maehara A, Mintz GS, Araki H, Choi SY, et al. (2010) Intravascular Ultrasound Classification of Plaque Distribution in Left Main Coronary Artery Bifurcations - Where Is the Plaque Really Located. Circ Cardiovasc Interv 3: 105-112.

5. Gruntzig AR, Senning A, Siegenthaler WE (1979) Non operative dilation of coronary artery stenosis: Percutaneous transluminal coronary angioplasty. N Engl J Med 301: 61-68.

6. Bentivoglio LG, VanRaden MJ, Kelsey SF, Detre KM (1984) Percutaneous transluminal coronary angioplasty (PTCA) in patients with relative contraindications: Results of the National Heart, Lung and Blood Institute PTCA registry. Am J Cardiol 53: 82C-88C.

7. Fajadet J, Chieffo A (2012) Current management of left main coronary artery disease. European Heart Journal 33:36-50.

8. Bing R, Yong ASC, Lowe HC (2015) Percutaneous Transcatheter Assessment of the Left Main Coronary Artery: Current Status and Future Directions. J Am Coll Cardiol Intv 8: 1529-1539.

9. Daniels DV, van't Veer M, Pijls NHJ, van der Horst A, Yong AS, et al. (2012) The Impact of Downstream Coronary Stenoses on Fractional Flow Reserve Assessment of Intermediate Left Main Disease. J Am Coll Cardiol Intv 5: 1021-1025.

10. Yong ASC, Daniels D, De Bruyne B, Kim HS, Ikeno F, et al. (2013) Fractional Flow Reserve Assessment of Left Main Stenosis in the Presence of Downstream Coronary Stenoses. Circ Cardiovasc Interv 6: 161-165.
11. Fearon WF, Yong AS, Lenders G, Toth GG, Dao C, et al. (2015) The Impact of Downstream Coronary Stenosis on Fractional Flow Reserve Assessment of Intermediate Left Main Coronary Artery Disease. J Am Coll Cardiol Intv 8: 398-403.

12. Pijls NHJ, van Son JA, Kirkeeide RL, De Bruyne B, Gould KL, et al. (1993) Experiment basis of determining maximum coronary, myocardial, and collateral blood flow by pressure measurements for assessing functional stenosis severity before and after percutaneous transluminal coronary angioplasty. Circulation 87: 1354-1367.

13. Pijls NHJ, De Bruyne B, Bech GJ, Liistro F, Heyndrickx GR, et al. (2000) Coronary pressure measurement to assess the hemodynamic significance of serial stenoses within one coronary artery: validation in humans. Circulation 102: 2371-2377.

14. Tonino PAL, De Bruyne B, Pijls NHJ, Siebert U, Ikeno F, et al. (2009) Fractional Flow Reserve versus angiography for guiding Percutaneous Coronary Intervention. N Engl J Med 360: 213-224.

15. Yaeger IA (2016) A multi-artery Fractional Flow Reserve (FFR) approach for handling coronary stenosis-stenosis interaction in the multi-vessel disease (MVD) arena. International Journal of Cardiology 203: 807-815.

16. Yaeger IA (2017) Resolution of All-stenotic 3-Artery Configurations in Percutaneous Coronary Intervention (PCI) Procedures by the Multiartery Fractional Flow Reserve (FFR) Method. Cardiovasc Investig 1:1-2.

17. Dodge JT, Brown BG, Bolson EL, Dodge HT (1992) Lumen Diameter of Normal Human Coronary Arteries - Influence of Age, Sex, Anatomic Variation, and Left Ventricular Hypertrophy or Dilation. Circulation 86: 232-246.

18. Yaeger IA (2015) Practicability of Multi-Artery Fractional Flow Reserve (FFR) Method in the Assessment of Some Stenotic Coronary Artery Configurations in Percutaneous Coronary Intervention (PCI) Procedures. Interv Cardiol J 2: 3-31.

19. Diken AI, Yalcinkaya A, Hanedan O, Gunertem E, Demir E, et al. (2017) Effect of tandem lesions on hemodynamic parameters: an experimental study. Kardiologia Polska 12: 1315-1323.

20.Wong JT, Le H, Suh WM, Chalyan DA, Mehraien T, et al. (2012) Quantification of fractional flow reserve based on angiographic image data. Int J Cardiovasc Imaging 28: 13-22.

21. De Bruyne B, Pijls NH, Heyndrickx GR, Hodeige D, Kirkeeide R, et al. (2000) Pressure-derived fractional flow reserve to assess serial epicardial stenoses: theoretical basis and animal validation. Circulation 101: 1840-1847. 